\title{
Tecnologias utilizadas pela enfermagem na prevenção de erros de medicação em pediatria
}

Recebido em: 08/05/2012

Aceito em: 12/07/2012

\author{
Márcia Maria Jordão ${ }^{1}$ \\ Michelini Fátima Silva² \\ Simone Vidal Santos ${ }^{3}$ \\ Nádia Chiodelli Salum ${ }^{4}$ \\ Sayonara Fátima F. Barbosa 5
}

Trata-se de revisão integrativa com objetivo de verificar quais tecnologias estão sendo utilizadas pela enfermagem para prevenir erros de medicação em pediatria. Realizou-se busca em bases de dados em saúde, de artigos nacionais e internacionais publicados entre os anos de 2006 e 2011. Os resultados mostram as estratégias utilizadas para minimizar erros de medicações, entre elas: padronização de medicamentos e drogas de alerta máximo, prescrições informatizadas, código de barras, dose unitária de medicamentos, dupla checagem, registros de enfermagem e participação do paciente na terapia. Alerta para a importância do uso de novas tecnologias que garantam maior segurança ao paciente pediátrico.

Descritores: Erros de Medicação, Prescrições de Medicamentos, Criança, Pediatria, Tecnologia.

\section{Technologies used by nursing preventing medication errors in pediatrics}

It is intelt is integrative review in order to verify which technologies are being used by nurses to prevent medication errors in pediatrics. Search was carried out on data bases in health, national and international articles published between 2006 and 2011. The results show the strategies used to minimize medication errors, including: the standardization of medicines and drugs of high alert, computerized prescriptions, bar coding, unit dose medication, double check, records of nursing and patient participation in therapy. It points to the importance of using new technologies to ensure greater safety for the pediatric patient.

Descriptors: Medication Errors, Drug Prescriptions, Children, Pediatrics, Technology.

\section{Tecnologías utilizadas por la enfermería en la prevención de errores de medicación en pediatría}

Se trata de una revisión integradora con el objetivo de verificar cuáles son las tecnologías que están siendo utilizadas por las enfermeras para prevenir errores de medicación en pediatría. Búsqueda se realizó en bases de datos en materia de salud, nacionales e internacional de artículos publicados entre los años 2006 y 2011. Los resultados muestran que las estrategias utilizadas para minimizar los errores de medicación, incluyendo: la estandarización de los medicamentos y las drogas de máxima alerta, las recetas informatizadas, códigos de barras, los medicamentos de dosis unitarias, doble control, los registros de participación de la enfermería y el paciente en la terapia. Señala la importancia de utilizar las nuevas tecnologías para garantizar una mayor seguridad para el paciente pediátrico Descriptores: Errores de Medicación, Recetas de Drogas, Los Niños, Pediatría, Tecnología.

\section{INTRODUÇÃO}

S egundo o National Coordinating Council for Medication Error Reporting and Prevention "um erro de medicação é qualquer evento evitável que pode causar ou induzir ao uso de medicação inadequada ou dano ao paciente enquanto a medicação está no controle do profissional de saúde, paciente ou consumidor"(1).

Cotidianamente, nos deparamos com erros de medicação, sejam eles de prescrição, dispensação, armazenamento, diluição e administração das drogas. Entende-se que esses aspectos não estão relacionados somente à equipe de enfermagem, mas ao conjunto da equipe de saúde.

Para gerar um sistema de medicação seguro, torna-se imprescindível qualidade e quantidade suficiente de recursos humanos, área física adequada, recursos financeiros, dispositivos e equipamentos com tecnologia apropriada, dentre outros ${ }^{(2)}$. As tecnologias auxiliam no aprimoramento do cuidado, entretanto,

1 Enfermagem - Mestrado Profissional - Gestão do Cuidado em Enfermagem/UFSC. Especialista em Enfermagem do Trabalho. Enfermeira da UTI Neonatal - Instituição: HU/ UFSC - Florianópolis-SC. Email: lajumar@hotmail.com

2 Mestranda do Mestrado Profissional - Gestão do Cuidado PEN/UFSC. Especialista em Gerontologia. Membro do Grupo de Pesquisa Saúde da Criança e do Adolescente GEPESCA - Enfermeira da Unidade de Pediatria - Instituição: HU/UFSC - Fpolis -SC.

3 Especialista em Estomaterapia. Enfermeira da Unidade de Pediatria - Instituição: HU/UFSC. Fpolis-SC.

4 Enfermeira Doutora em Filosofia, Saúde e Sociedade. Docente do Mestrado Profissional Gestão do Cuidado em Enfermagem/UFSC, Membro do Grupo de pesquisa GIATE/ UFSC, Coordenadora do Centro de Educação e Pesquisa em Enfermagem/DE/HU/UFSC.

5 Enfermeira Doutora em Enfermagem. Docente do Curso de Pós-Graduação do Departamento de Enfermagem da UFSC, Sub-coordenadora do Grupo de Pesquisa GIATE.

*Texto produzido na disciplina: Tecnologias Emergentes para Educação, Pesquisa e Cuidado em Saúde e Enfermagem - Mestrado Profissional - Gestão do Cuidado em

Enfermagem/UFSC. 
não se pode esquecer que é o cuidado que utiliza a tecnologia para garantir um cuidado de enfermagem mais eficiente, eficaz e convergente aos requerimentos do ser cuidado(3).

Como enfermeiras que trabalham diretamente com crianças, entendemos que o cuidado deve ser redobrado, haja vista as pequenas doses utilizadas em pediatria. A disponibilidade de diferentes formas farmacêuticas do mesmo medicamento, dosagem incorreta, falta de posologia padronizada e imaturidade dos órgãos e sistemas são fatores que tornam os pacientes pediátricos mais suscetíveis a erros de medicação e a potenciais complicações decorrentes da administração de medicamentos ${ }^{(4)}$.

Diante desse contexto, optou-se por realizar uma revisão integrativa, com o objetivo de verificar que tecnologias estão sendo utilizadas pela enfermagem para prevenir os erros de medicação em pediatria.

\section{METODOLOGIA}

Trata-se de uma revisão integrativa da literatura de publicações indexadas nas bases de dados Medline, Lilacs e Scielo. A coleta de dados ocorreu nos meses de abril e maio de 2011. Como critérios de inclusão para a seleção da amostra, utilizaram-se publicações com textos completos, nacionaiseinternacionais, entre os anos de 2006 e 2011, que abordavam as palavras "error", "medication" e "children" e os descritores "Erros de Medicação", "Criança", "Pediatria", "Prescrições de Medicamentos" e "Tecnologia". Refinandose primeiramente com os descritores "Erros de Medicação" X "Criança”, posteriormente com "Pediatria" X "Prescrições de Medicamentos" e finalmente com "Erros de Medicação" X "Tecnologia".

\section{RESULTADOS E DISCUSSÃO}

Considerando a base de dados Medline, foram encontrados 546 artigos e/ou resumos, dentre os quais apenas 15 estavam adequados aos critérios de inclusão. Na base de dados Scielo foram encontrados cinco artigos e/ou resumos, no entanto, nenhuma publicação atendeu os critérios de inclusão. Na base de dados Lilacs, encontraram-se cinco artigos e/ou resumos, sendo que apenas três atenderam os critérios de inclusão dessa pesquisa, e um se apresentava repetido.

Ao analisar os dados, pode-se observar que a maior parte dos artigos, $29,4 \%$ (5), foi publicada no ano de $2006,17,6 \%$ (3) em 2007, 23,5\% (4) em 2008, 23,5\% (4) em 2009 e 5,9\% (1) em 2010. Esses dados provavelmente estão relacionados à ênfase dada ao assunto em 2004, quando a Organização Mundial de Saúde (OMS) lançou a Aliança Mundial para Segurança do Paciente, com o intuito de reunir recomendações destinadas a garantir a segurança dos pacientes em nível mundial(5).

Quanto ao idioma da publicação, 82,3\% (14) estavam disponíveis em inglês, $11,7 \%$ (2) em português e 5,9\% (1) em espanhol. Isso denota a pequena quantidade de estudos referentes ao tema realizados em nosso país e acredita-se que esse fato esteja relacionado à falta de cultura institucional de registro de notificação de erros e ao fato de a segurança do paciente estar em evidência há pouco tempo no Brasil.

Em relação aos erros de medicação, os de prescrição são os mais comuns, sendo os antimicrobianos e as dosagens erradas das drogas os itens mais frequentemente associados com os erros $^{(6)}$.

Quanto ao tipo de tecnologia utilizada pela enfermagem, o estudo apontou que apenas $41,1 \%$ (7) realmente utilizaram algum tipo de tecnologia, sendo que em sua maioria - 17,6\% (3) - as prescrições são computadorizadas, 5,9\% (1) padronização de concentração de drogas em um hospital pediátrico, 5,9\% (1) sistema computadorizados para notificação de erros, 5,9\% (1) a comunicação médico/paciente e comunicação interprofissional e $5,9 \%$ (1) roteiro deações para administração de medicamentos. Nos demais artigos, em 58,9\% (10) encontram-se apenas recomendações de estratégias, a maioria baseada no Food and Drug Administration (FDA) e Joint Commission on Accreditation of Healthcare Organizations (JCAHO), que podem ser utilizadas para prevenir erros de medicação em pediatria.

Com relação ao uso de tecnologias, $47 \%$ (8) dos estudos indicam o uso de prescrições informatizadas como ferramenta importante, com obtenção de resultados comprovadamente satisfatórios para a segurança do paciente. Acredita-se que a prescrição médica informatizada pode auxiliar na prevenção de erros, pois facilita a leitura e o entendimento do que está prescrito, tendo em vista a ilegibilidade da escrita de muitos profissionais ${ }^{(7)}$. 5,9\% (1) dos estudos incluem o apoio à decisão médica informatizada, que auxilia na prescrição da droga a ser utilizada, apresentando melhoria na segurança do paciente ${ }^{(7)}$. Os sistemas de apoio à decisão médica informatizada possibilitam integrar informações farmacêuticas compreensivas e confiáveis, com acesso aos dados acerca das possíveis interações ou efeitos adversos dos medicamentos, habilitando os profissionais a promover medicação mais adequada aos pacientes e simplificando a administração das prescrições ${ }^{(8)}$.

O programa informatizado para concentração de drogas é outra tecnologia descrita em $11,7 \%$ (2) dos estudos e $23,5 \%$ (4) sugerem a padronização de administração de drogas de alerta máximo. Outras tecnologias utilizadas incluem a utilização de tabelas para conversão da dose de medicamentos em 5,9\% (1) dos estudos e uso de monitores fisiológicos como ferramenta para detectar reações adversas ou supersedação em crianças anestesiadas em um estudo 5,9\%(9).

Ressalta-se a importância de os profissionais de saúde receberem formação adequada para o uso de tecnologias, administração, monitoração e reanimação de pacientes, 
conforme consta em 5,9\% (1) dos estudos. A recomendação para capacitação sobre farmacologia aparece em 17,6\% (3) dos artigos.

A indicação da necessidade de padronização dos medicamentos em cada instituição foi descrita em 17,6\% (3) dos estudos e o programa de gestão de antimicrobianos, que auxilia o médico na prescrição do correto antimicrobiano, foi encontrada em $5,9 \%^{(1)}$. A utilização do código de barras para dispensação e checagem dos medicamentos foi encontrada em $11,7 \%^{(2)}$ dos artigos. O código de barras pode ser utilizado como recurso em pediatria e neonatologia, utilizando programas com capacidade de fornecer etiquetas com código legível para pequenos volumes e dose específica para cada paciente ${ }^{(9)}$. O uso de dose unitária de medicamentos, dispensação da dose exata para cada paciente, foi encontrada em $11,7 \%^{(2)}$ dos estudos.

A rotulagem clara e fácil foi descrita como importante estratégia para evitar erros de medicação em $23,5 \%^{(4)}$ dos artigos. O FDA recomenda medidas para melhorar a rotulagem dos medicamentos pediátricos disponíveis. Essas medidas devem incluir linguagem fácil e proibição de gráficos enganosos sobre todas as embalagens $^{(10)}$. Como ferramenta para prevenção de erros na administração de medicamentos, sugere-se o uso de seringas orais, ou seja, seringas específicas para administração de medicamentos por via oral, que se apresentam com cores e formatos diferentes das utilizadas em administração parenteral. Estas foram evidenciadas em 5,9\% (1) dos estudos.

Constatou-se que $17,6 \%{ }^{(3)}$ dos artigos identificam a dupla checagem como importante ferramenta para assegurar o correto procedimento. Nesse processo, dois profissionais verificam se a prescrição, o preparo e a administração do medicamento estão corretos. A ferramenta de "volta da leitura", em que o profissional repete o que está sendo solicitado, assegurando a correta compreensão da informação, esteve elencado em 5,9\% (1) dos artigos. De acordo com 5,9\% (1) dos estudos, as prescrições verbais devem ser desencorajadas e, quando utilizadas, medidas de segurança devem ser implementadas como repetir em voz alta, de modo completo, a informação dada pelo emissor ${ }^{(11)}$. Segundo as normas da $\mathrm{JCAHO}$, as prescrições realizadas por telefone devem ser reservadas para inclusões, alterações, ou suspensões de itens. E, "ao receber a ordem telefônica, o profissional terá que escrever e ler de volta para o médico para que ele confirme a informação transmitida. Tais itens devem ser validados pessoalmente pelo médico em até $24 \mathrm{~h}^{\prime \prime}(12: 1)$.

Com relação aos registros de enfermagem sobre a administração dos medicamentos, $11,7 \%$ (2) descrevem que devem ser preenchidos corretamente, especificando rotineiramente o procedimento e a aceitação do paciente à terapêutica. 5,9\% (1) dos artigos relatam que deve ser elaborado, pela equipe de saúde, relatório completo da internação contendo, entre outras informações, os medicamentos utilizados e ocorrência de reações adversas ou transferência para outra instituição(9).

Verificou-se em 17,6\% (3) dos estudos que a comunicação entre profissional, paciente e família é uma importante estratégia para prevenir erros de medicação. Segundo a JCAHO, a comunicação ineficaz é uma das principais causas de eventos sentinelas, considerando que a comunicação eficaz pode reduzir os erros e melhorar a segurança de todos os envolvidos ${ }^{(13)}$. Quanto às informações dadas à família, deve-se utilizar linguagem clara e confirmar a compreensão dos pais acerca do uso dos medicamentos, através de técnicas como retorno do que foi ensinado ${ }^{(14)} .17,6 \%$ (3) dos estudos revelam ser fundamental a participação ou maior aproximação do farmacêutico junto à equipe de atendimento ao paciente.

Umestudo (5,9\%) recomendou variadas ferramentas como:uso de dispositivos dosadores automatizados, formulário para gestão de assistência pediátrica, ambiente adequado e pessoal competente na farmácia, farmacêutico disponível, melhora na qualidade dos relatórios de erros e revisão da avaliação do uso de drogas e medicamentos, acesso dos profissionais de saúde a informações clínicas e referências atuais, sistemas de alerta para detecção de eventos adversos no computador, uso de software farmacêutico, sistemas baseados em mistura de intravenosos na farmácia, padronização de equipamentos e incentivo às reuniões da equipe de saúde para revisão das decisões ${ }^{(15)}$. Apontam que o conjunto dessas estratégias minimiza os eventos adversos, contribuindo para a segurança do paciente.

Reforçando a necessidade de notificação de erros, 5,9\% (1) dos estudos utilizaram a base computadorizada para esse fim, demonstrando eficácia do sistema, tendo em vista a confiabilidade e rapidez com que os dados foram contabilizados.

A JCAHO define alguns critérios para favorecer a segurança na administração de medicamentos como a necessidade de haver fontes de informações sobre fármacos disponíveis para os profissionais envolvidos com o uso de medicamentos; a prescrição, a requisição e a transcrição de medicamentos devem ser orientadas por políticas e procedimentos, incluindo ações para diminuir a ilegibilidade, e os profissionais devem ser capacitados para essas práticas; constar no prontuário de cada paciente uma lista dos medicamentos prescritos ou solicitados, assim como informações sobre dosagens e horários em que a medicação foi administrada, incluindo os medicamentos administrados "se necessário"; as prescrições devem ser revisadas antes da dispensação quanto a: pertinência da droga, frequência, dose e via de administração; duplicidade terapêutica; sensibilidades ou alergias; interações entre medicamentos, e entre medicamentos e alimentos; peso do paciente; em caso de dúvidas, deve-se entrar em contato com 
o profissional que prescreveu ou solicitou o medicamento; os medicamentos devem ser dispensados da forma mais pronta possível para administração e etiquetados adequadamente; as medicações, doses dos medicamentos e vias de administração devem ser conferidas com a prescrição ou requisição; os medicamentos devem ser administrados na hora certa e da forma prescrita, devendo ser anotados no prontuário do paciente; devem ser monitorados os efeitos dos medicamentos nos pacientes, incluindo efeitos adversos e quase falhas ${ }^{(16)}$.

\section{CONSIDERAÇÕES FINAIS}

Os dados evidenciaram a existência de uma variedade de tecnologias que podem ser utilizadas pelos profissionais de saúde na prevenção de erros de medicação em pediatria. As tecnologias mais utilizadas/recomendadas foram: a padronização dos medicamentos dentro das instituições, o uso de prescrições informatizadas, programa informatizado para concentração de drogas, padronização de administração de drogas de alerta máximo, capacitação em farmacologia, uso de código de barras, dose unitária de medicamentos, rotulagem clara e fácil, dupla checagem, completude dos registros de enfermagem, melhora da comunicação, participação do paciente em sua terapia. Entretanto, observa-se que ainda há poucas instituições que têm implantadas tecnologias e métodos para garantir a segurança do paciente pediátrico de forma sistemática. Observa-se que, mesmo em países desenvolvidos, onde existem hospitais de grande porte, que deveriam utilizar tecnologias como recurso para favorecer a segurança do paciente, a realidade encontrase ainda muito distante da situação ideal. Talvez esse fato esteja relacionado aos custos elevados na implantação de tecnologias e em todas as demais necessidades em que se exige capacitação de equipes e manutenção dos sistemas e equipamentos.

Nesse contexto, constata-se que cuidados inseguros de saúde resultam em expressiva morbidade e mortalidade evitáveis, gastos adicionais com a manutenção dos sistemas de saúde e representam uma grande preocupação na atualidade ${ }^{(17)}$. Nesse sentido, acredita-se que os custos se tornam mínimos, em detrimento das consequências ocasionadas por erros relacionados à administração de medicamentos, que poderiam ser minimizados com o uso de tecnologias. A responsabilidade pelo cuidado em saúde é de toda a equipe de saúde, visto que tanto o paciente pediátrico quanto sua família vivem um momento de fragilidade, deixando-se conduzir pelo profissional e confiando a ele sua saúde e bem-estar.

Os resultados obtidos nessa revisão reforçam a importância de desenvolver indicadores de segurança do paciente, com base na melhor evidência científica disponível, e de os adaptar à realidade de cada país para garantir sua viabilidade.

\section{Referências}

1. National Coordinating Council for Medication Error Reporting and Prevention [Internet]. Disponivel no site: http://www.mccmerp.org . Acesso em 05/05/2011. 2. Coimbra, JAH. Prevenção e detecção de erros de medicação. Revista Ciência, Cuidado e Saúde. 2006, 5(Supl.): 142- 48.

3. Rocha, PK; Prado, ML; Wal, ML; Carraro, TE. Cuidado e tecnologia: aproximações através do modelo de cuidado. Rev. Bras Enferm 2008, 61(1):113-6.

4. Gonzales, K. Medication administration error and the pediatric population: a systematic search of the literature. Journal of Pediatric Nursing. 2010, 25: 555-65. 5. Silva, AEBC. Segurança do paciente: desafios para a prática e a investigação em enfermagem. Revista Eletrônica de Enfermagem [Internet]. 2010, 12(3):422. Disponível em: http://www.fen.ufg.br/revista/v12/n3/v12n3a01.htm. Acesso em 08/06/2011. 6. Lewis, PJ; Dornan, T; Taylor, D; Tully, MP; Wass, V; Ashcroft, DM. Prevalence, incidence and nature of prescribing errors in hospital inpatients: a systematic review. Drug Safety 2009, 32(5): 379-89

7. Calligaris, L; Panzera, A; Arnoldo, L; Londero, C; Quattrin, R; Troncon,MGB. et al. Errors and omissions in hospital prescriptions: a survey of prescription writing in a hospital. BMC Clinical Pharmacology 2009, 9(9). Disponivel no site: http://www. biomedcentral.com/1472-6904/9/9. Acesso em 05/05/2011.

8. Tomé, A; Broeiro, P; Faria-Vaz, A. Os sistemas de prescrição electrônica. Rev Port Clin Geral 2008; 24:632-40.

9. Broussard, L. Small size, big risk: preventing neonatal and pediatric medication errors. Nursing for Women's Health 2010, 4: 405-8.

10. Lokker, N; Sanders, L; Perrin, EM; Kumar, D; Finkle, J; Franco, V; et al. Parental misinterpretations of over-the-counter pediatric cough and cold medication labels. Pediatrics 2009, 123(6): 1464-71.
11. Coren. Conselho Regional de Enfermagem do Estado de São Paulo. Rede Brasileira de Enfermagem e Segurança do Paciente. Polo São Paulo. 10 Passos para a segurança do paciente, 2010.

12. Informativo Albert Einstein - Sociedade Beneficente Israelita Brasileira Joint Commission International. [Internet] Edição especial. Março, 2009. Disponível no site: www.medicalsuite.einstein.br/informativos_pdf/edicao_06_ especial_jci.pdf. Acesso em 27/06/2011

13. Morgan, N. Opportunities for performance improvement in relation to medication administration during pediatric stabilization. Qual Saf Health Care 2006, 15(3):179-83.

14. Bokhour, BG. Patterns of concordance and non-concordance with clinician recommendations and parents' explanatory models in children with asthma. Patient Education and Counseling 2008, 70(3); 376-85.

15. Miller, MA; Robinson, KA; Lubomski, LH; Rinke, ML; Pronovost, PJ. Medication errors in paediatric care: a systematic review of epidemiology and an evaluation of evidence supporting reduction strategy recommendations. Qual Saf Health Care 2007, 16: 116-26.

16. Padrões de Acreditação da Joint Commission International para Hospitais [editado por]

Consórcio Brasileiro de Acreditação de Sistemas e Serviços de Saúde - Rio de Janeiro:CBA: 2010. 288p.

17. World Health Organization/World Alliance for Patient Safety. Summary of the evidence on patient safety: implications for research. The Research Priority Setting Working Group of the World Alliance for Patient Safety. Geneva: World Health Organization; 2008. 
QUEM AMPLIA SEUS CONHECIMENTOS AUMENTA SUAS POSSIBILIDADES NA PROFISSÃO.

Cursos Gratuitos de Enfermagem Via Internet

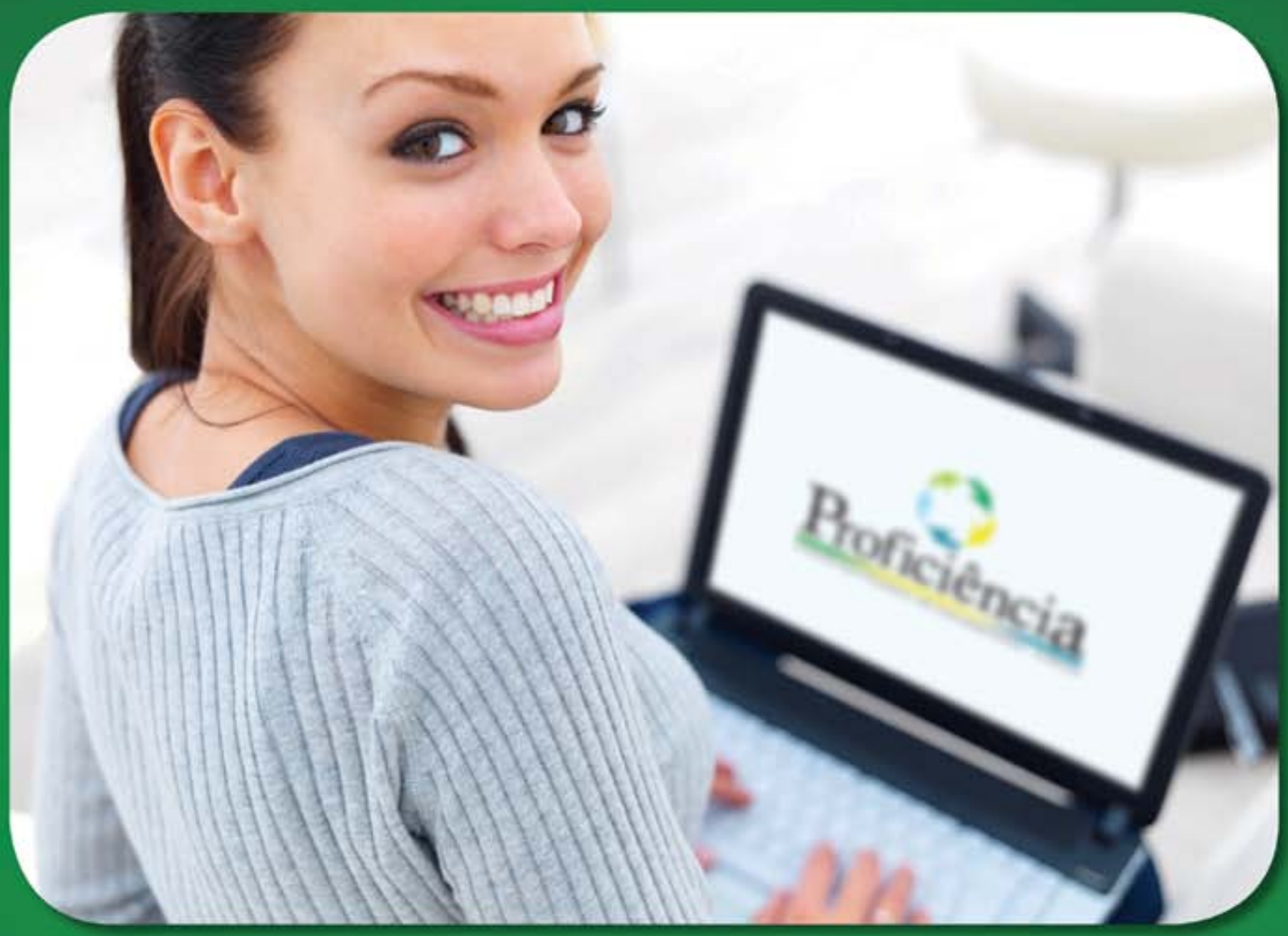

Para Profissionais de Enfermagem (Enfermeiros, Técnicos e Auxiliares) que estiverem REGULARMENTE INSCRITOS no Conselho Regional de seu Estado.

Informações através do site: www.programaproficiencia.com.br

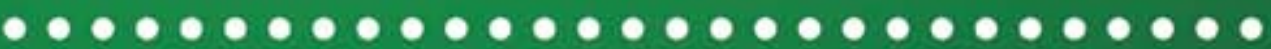

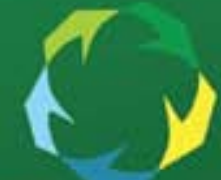

